\title{
Seleção de matrizes e clones de mangabeira para o cultivo in vitro
}

\author{
Luana de Lima Machado( ${ }^{(1)}$, Maria Lucrécia Gerosa Ramos ${ }^{(2)}$, Linda Styer Caldas ${ }^{(3)}$ e Lúcio José Vivaldi(4) \\ ${ }^{(1)}$ Faculdade da Terra de Brasília, Av. Recanto das Emas, Qd. 203, Lt. 32, CEP 72610-300 Recanto das Emas, DF. E-mail: \\ Luana@ftb.edu.br ${ }^{(2)}$ Universidade de Brasília (UnB), Fac. de Agronomia e Medicina Veterinária, Caixa Postal 04508, CEP 70910-970 \\ Brasília, DF. E-mail: lucrecia @ unb.br ${ }^{(3)} \mathrm{UnB}$, Dep. de Botânica. E-mail: lindasc @ terra.com.br ${ }^{(4)}$ UnB, Dep. de Estatística. \\ E-mail: vivaldi@unb.br
}

\begin{abstract}
Resumo - Altas taxas de mortalidade em viveiro de mudas de mangabeira (Hancornia speciosa) impedem seu uso na reversão do processo de degradação das terras e na manutenção da produtividade e integridade ambiental do Cerrado. O objetivo deste trabalho foi selecionar matrizes e clones, provenientes de propagação sexuada e assexuada, com potencial de propagação in vitro, para produção de mudas de mangabeira. Foram coletados frutos de 11 matrizes e de cada matriz selecionaram-se 24 sementes em bom estado fitossanitário. Após a desinfecção, as sementes foram inoculadas em meio MS, sem reguladores de crescimento, obtendo-se uma média de germinação de $92,4 \%$, e as matrizes não apresentaram diferença significativa entre si. Na fase de multiplicação, em meio MS, com os reguladores de crescimento BAP (6-benzilaminopurina) e AIB (ácido indol3-butírico), ambos na concentração de $1,28 \mathrm{mg} \mathrm{L}^{-1}$, a melhor matriz foi a $\mathrm{C} 1$ e o melhor clone foi o $\mathrm{C} 115$. Em todas as fases foi observada alta variabilidade, em menor porcentagem na matriz e maior porcentagem no clone dentro da matriz. A seleção deve ser realizada principalmente nos clones dentro da matriz.
\end{abstract}

Termos para indexação: Hancornia speciosa, micropropagação, germinação in vitro, fruticultura tropical.

\section{Selection of parents and clones of mangabeira for in vitro cultivation}

\begin{abstract}
High tax of mortality of mangabeira (Hancornia speciosa) seedlings in greenhouse conditions hinders its use on the reversion of the degradation of the natural vegetation, as well as on the preservation of the production and environmental integrity. The objective of this work was to select better mother plants and clones from sexual and assexual propagation, with potential to survive in vitro, for producing mangaba seedlings. Fruits were collected from 11 mother plants and from each plant, 24 seeds without aparent diseases were selected. After superficial desinfection, seeds were inoculated in MS medium without growth regulators and having a germination of $92.4 \%$ without significant difference between mother plants. On multiplication phase, with MS medium, with growth regulators BAP (6-benzilaminopurine) and IBA (indol-3-butiric acid), both at concentration of $1.28 \mathrm{mg} \mathrm{L}^{-1}$; the better mother plant was $\mathrm{C} 1$ and better clone was $\mathrm{C} 115$. During all phases of this work there was a high variability, mainly among clones. The selection must be made mainly among clones in mother plants.
\end{abstract}

Index terms: Hancornia speciosa, micropropagation, germination in vitro, tropical fruticulture.

\section{Introdução}

O Cerrado é considerado a savana de maior biodiversidade vegetal do mundo, com uma área de 204 milhões de hectares, distribuídos principalmente nos Estados de Minas Gerais, Goiás, Mato Grosso, Mato Grosso do Sul, Tocantins, Bahia, Piauí, Maranhão e Distrito Federal (Pereira et al., 2001).

Todavia, a expansão das áreas agricultáveis e o seu mau uso, com monoculturas, o desmatamento e o extrativismo predatório, estão destruindo o Cerrado. Para reverter o processo de degradação das terras, com a manutenção da produtividade e integridade ambiental, sugere-se a exploração econômica das fruteiras nativas, que têm grande potencial, por causa do aproveita- mento alimentar, proteção ambiental e florestal, controle da erosão e a conservação de nascentes de rios e lagos, bem como proteção e conservação de espécies animais e vegetais em vias de extinção (Melo, 1999).

A mangabeira (Hancornia speciosa) possui um fruto extremamente apreciado que é consumido in natura, além de ser utilizado na fabricação de refrescos, sorvetes, doces secos, compotas, no preparo de vinho e vinagre (Parente et al., 1985) e na medicina popular (Ferreira, 1980). Além do aproveitamento da fruta, a mangaba pode ser explorada na produção de borracha (Paula, 1992), que é utilizada na região dos cerrados para impermeabilizar tecidos, confeccionar bolas e para tratar luxações e hipertensão (Hirschmann \& Arias, 1990). 
No Cerrado, a mangabeira ocorre principalmente em encostas pedregosas. As doenças que a acometem são a mancha-das-folhas, causada por Pseudocercospora sp., e a podridrão-de-raiz, causada por Cylindrocladium clavatum (Junqueira et al., 1997). Essas doenças dificultam a germinação das sementes e a obtenção de mudas sadias que se estabeleçam nos viveiros, tornando-se um entrave para a sua expansão e plantio comercial (Andrade et al., 1999).

O cultivo comercial pode ser viabilizado em menos tempo pelo processo de micropropagação. A obtenção dos explantes, a partir de plântulas de sementes germinadas in vitro, evita a contaminação do material vegetal e a baixa resposta morfogenética dos tecidos arbóreos adultos (Grigoletto, 1997).

O objetivo deste trabalho foi selecionar matrizes e clones, provenientes de sementes, com potencial de propagação in vitro, para produção de mudas de mangabeira.

\section{Material e Métodos}

Foram coletados frutos provenientes de onze matrizes de mangabeira em várias localidades descritas a seguir, entre outubro de 2000 e abril de 2001. A posição geográfica de cada matriz foi identificada com o auxílio de um aparelho de GPS (sistema de posicionamento global).

Foram coletados frutos de quatro matrizes, em Pirenópolis, GO, que receberam as denominações de $\mathrm{P} 1, \mathrm{P} 8, \mathrm{P} 12$ e P13. Os frutos tinham tamanho e peso variados e possuíam coloração verde, característica da espécie. Na Reserva Ecológica do Gama (DF) foram coletados frutos de mangabeira, em uma matriz que recebeu a denominação de G1, com tamanho reduzido e bem uniformes, apresentando coloração verde. No PADF (Produtores Agrícolas do Distrito Federal), foram coletados frutos de cinco matrizes, denominadas de $\mathrm{C} 1, \mathrm{C} 2, \mathrm{C} 3, \mathrm{C} 4$ e C5. A casca dos frutos era de coloração avermelhada. Os frutos apresentavam tamanho variado e possuíam manchas características de ataque de Colletotrichum gloeosporioides (Muniz et al., 1997). Os frutos coletados na Reserva Ecológica de Águas Emendadas (DF) de uma matriz, A1, eram uniformes e de tamanho reduzido.

Após a coleta, os frutos foram mantidos a $4^{\circ} \mathrm{C}$ por aproximadamente 15 horas e, para cada matriz, foram despolpados e misturados. As sementes de cada fruto foram homogeneizadas e aquelas contaminadas ou inviáveis foram eliminadas. As consideradas viáveis foram separadas aleatoriamente em 24 sementes por matriz. Em seguida, as sementes foram lavadas com água destilada e o seu tegumento removido, em câmara de fluxo laminar, com o auxílio de um bisturi e novamente lavadas com água destilada. A desinfestação superficial das sementes foi feita com imersão em álcool 70\%, por um minuto e, em seguida, em solução de hipoclorito de sódio a $1 \%$ por cinco minutos e lavadas dez vezes com água destilada. Posteriormente, foram inoculadas em meio MS, sem a adição de reguladores de crescimento, com $5 \mathrm{~g} \mathrm{~L}^{-1}$ de ágar e $\mathrm{pH}$ ajustado a 5,8. O meio foi distribuído em tubos de ensaio $(25 \times 150 \mathrm{~mm})$, com $15 \mathrm{~mL}$ por tubo, que, a seguir, foram vedados com tampas de plástico e esterilizados em autoclave por $17 \mathrm{mi}$ nutos após a temperatura atingir $127^{\circ} \mathrm{C}$, sob $1,5 \mathrm{~atm}$ de pressão. Após a inoculação, foram transferidas para a sala de crescimento, com intensidade luminosa de 20 a 50 ì moles $\mathrm{m}^{-2} \mathrm{~s}^{-1}$, temperatura em torno $24^{\circ} \mathrm{C}$ e um fotoperíodo de 16 horas. Após cinco semanas, avaliouse a germinação das sementes, calculando-se a porcentagem de germinação.

No processo de multiplicação, foi utilizado o meio nutritivo sólido MS, suplementado com BAP (6-benzilaminopurina) e AIB (ácido indol-3-butírico), ambos na concentração de $1,28 \mathrm{mg} \mathrm{L}^{-1}$ (Grigoletto,1997). As plântulas oriundas da germinação das sementes foram divididas em segmentos nodais e apicais, sendo realizadas nove repicagens e a sobrevivência dos explantes avaliada na primeira, terceira, quinta, sétima e nona repicagem.

Os dados obtidos foram analisados pelo programa estatístico do SAS Institute (1985). O experimento foi analisado como inteiramente casualizado e o número de repetições variou de acordo com o número de clones obtidos. O processo de geração das observações induziu à utilização do modelo: $y_{i j}=i ̀ ~+a_{i}+e_{i j}$, em que $y_{i j}$ é o valor de uma variárel; ì é a média geral; $a_{i}$ é o efeito da matriz e $\mathrm{e}_{\mathrm{ij}}$ é o erro do modelo. A parcela foi a matriz e o número de repetições variou para cada matriz. A análise de variância utilizada foi o procedimento denominado modelo linear geral (GLM) e as médias comparadas pelo teste de Tukey, a 5\% de probabilidade. $\mathrm{Na}$ análise não-paramétrica, utilizaram-se os escores de Wilcoxon (Wolf \& Hollander, 1973). Os testes de comparação múltipla, e as comparações dos escores dos tratamentos foram analisados pelo teste de KruskalWallis, aproximação de qui-quadrado. 


\section{Resultados e Discussão}

Foi obtida germinação média de sementes entre as matrizes de $92,41 \%$, com baixa taxa de contaminação, indicando a eficiência do tratamento utilizado para a desinfecção das sementes.

Comparando-se os resultados obtidos pela análise de variância na germinação, não foi observada diferença significativa entre as 11 matrizes, podendo-se atribuir apenas 3\% da variância devido às matrizes e $97 \%$ devido aos clones dentro da matriz (Tabela 1). Na análise não-paramétrica, não se obteve diferença significativa entre as matrizes na germinação (Tabela 2).

Nas etapas seguintes, foram feitas nove repicagens dos clones obtidos, a partir das sementes das 11 matrizes. Na primeira multiplicação dos explantes, houve di-

Tabela 1. Componentes de variância de matrizes e clones na germinação de sementes de mangabeira, após 30 dias de incubação.

\begin{tabular}{|c|c|c|c|c|c|c|}
\hline \multirow[t]{2}{*}{ Variável $^{(1)}$} & \multirow[t]{2}{*}{ Germinação } & \multicolumn{5}{|c|}{ Repicagem } \\
\hline & & $1^{\mathrm{a}}$ & $3^{\mathrm{a}}$ & $5^{\mathrm{a}}$ & 7 a & $9^{\text {a }}$ \\
\hline$\hat{\sigma}_{M}^{2}$ & 0,0023 & 1,41 & 4,189 & 20,49 & 78,41 & $1.390,41$ \\
\hline$\hat{\sigma}_{\mathrm{C}}^{2}$ & 0,068 & 2,15 & 15,66 & 43,71 & 329,32 & 10.359 \\
\hline$\hat{\sigma}_{\mathrm{M}(\%)}^{2}$ & 3 & 39 & 21 & 32 & 19 & 12 \\
\hline$\hat{\sigma}_{\mathrm{C}(\%)}^{2}$ & 97 & 61 & 79 & 68 & 81 & 88 \\
\hline
\end{tabular}

Tabela 2. Ordenação realizada pela análise não-paramétrica na germinação das sementes de mangabeira, originárias das matrizes estudadas ${ }^{(1)}$.

\begin{tabular}{lrrrrrr}
\hline Matriz & \multirow{2}{*}{ Germinação } & \multicolumn{5}{c}{ Repicagem } \\
\cline { 3 - 7 } & & \multicolumn{1}{c}{$1^{\mathrm{a}}$} & \multicolumn{1}{c}{ a $^{\mathrm{a}}$} & \multicolumn{1}{c}{$5^{\mathrm{a}}$} & $7^{\mathrm{a}}$ & $9^{\mathrm{a}}$ \\
\hline P12 & $142,5 \mathrm{a}$ & $173,5 \mathrm{a}$ & $187,5 \mathrm{a}$ & $125,6 \mathrm{~b}$ & $112,0 \mathrm{~b}$ & $119,0 \mathrm{~b}$ \\
C1 & $142,5 \mathrm{a}$ & $173,5 \mathrm{a}$ & $165,5 \mathrm{a}$ & $209,2 \mathrm{a}$ & $183,1 \mathrm{a}$ & $201,4 \mathrm{a}$ \\
C3 & $142,5 \mathrm{a}$ & $173,5 \mathrm{a}$ & $165,5 \mathrm{a}$ & $203,0 \mathrm{a}$ & $138,7 \mathrm{a}$ & $130,0 \mathrm{~b}$ \\
C4 & $142,5 \mathrm{a}$ & $173,5 \mathrm{a}$ & $165,5 \mathrm{a}$ & $194,3 \mathrm{a}$ & $144,9 \mathrm{a}$ & $135,7 \mathrm{a}$ \\
C2 & $137,0 \mathrm{a}$ & $151,5 \mathrm{a}$ & $160,0 \mathrm{a}$ & $139,3 \mathrm{a}$ & $145,4 \mathrm{a}$ & $135,8 \mathrm{a}$ \\
G1 & $131,5 \mathrm{a}$ & $102,0 \mathrm{~b}$ & $123,4 \mathrm{a}$ & $97,8 \mathrm{~b}$ & $118,0 \mathrm{a}$ & $124,6 \mathrm{~b}$ \\
C5 & $131,5 \mathrm{a}$ & $124,0 \mathrm{a}$ & $110,3 \mathrm{~b}$ & $101,7 \mathrm{~b}$ & $112,0 \mathrm{~b}$ & $119,0 \mathrm{~b}$ \\
P1 & $126,0 \mathrm{a}$ & $124,0 \mathrm{a}$ & $142,1 \mathrm{a}$ & $105,5 \mathrm{~b}$ & $112,0 \mathrm{~b}$ & $119,0 \mathrm{~b}$ \\
P8 & $120,5 \mathrm{a}$ & $74,5 \mathrm{~b}$ & $79,2 \mathrm{~b}$ & $79,0 \mathrm{~b}$ & $112,0 \mathrm{~b}$ & $119,0 \mathrm{~b}$ \\
P13 & $120,5 \mathrm{a}$ & $63,5 \mathrm{~b}$ & $56,5 \mathrm{~b}$ & $79,0 \mathrm{~b}$ & $112,0 \mathrm{~b}$ & $119,0 \mathrm{~b}$ \\
A1 & $120,5 \mathrm{a}$ & $124,0 \mathrm{a}$ & $101,3 \mathrm{~b}$ & $123,2 \mathrm{~b}$ & $167,4 \mathrm{a}$ & $134,8 \mathrm{a}$ \\
\hline
\end{tabular}

${ }^{(1)}$ Em cada coluna, médias seguidas da mesma letra não diferem entre si a $5 \%$ de probabilidade, pelo teste de Wilcoxon. ferença significativa entre as matrizes, atribuindo-se 39\% da variabilidade à matriz e $61 \%$ aos clones dentro da matriz (Tabela 1).

Houve grande variação entre as matrizes na porcentagem de sobrevivência na primeira avaliação (Figura 1). A menor porcentagem de sobrevivência dos clones foi observada na matriz P13 (20\%) e a maior nas matrizes $\mathrm{P} 12, \mathrm{C} 1, \mathrm{C} 3$ e C4 (100\%).

Tomando-se por base a análise não-paramétrica, pode-se separar as matrizes em dois grupos diferenciados; o primeiro com as matrizes $\mathrm{P} 12, \mathrm{C} 1, \mathrm{C} 3, \mathrm{C} 4, \mathrm{C} 2$, $\mathrm{C} 5, \mathrm{P} 1$ e A1 e um segundo contendo as matrizes G1, P8 e P13, com os escores menores que aqueles do primeiro grupo (Tabela 2).

$\mathrm{Na}$ terceira repicagem dos clones, houve diferença significativa entre as matrizes, atribuindo-se $21 \%$ da variabilidade à matriz e $79 \%$ ao clone dentro da matriz (Tabela 1).

Comparando-se com as repicagens anteriores, houve um aumento da variabilidade em virtude do clone
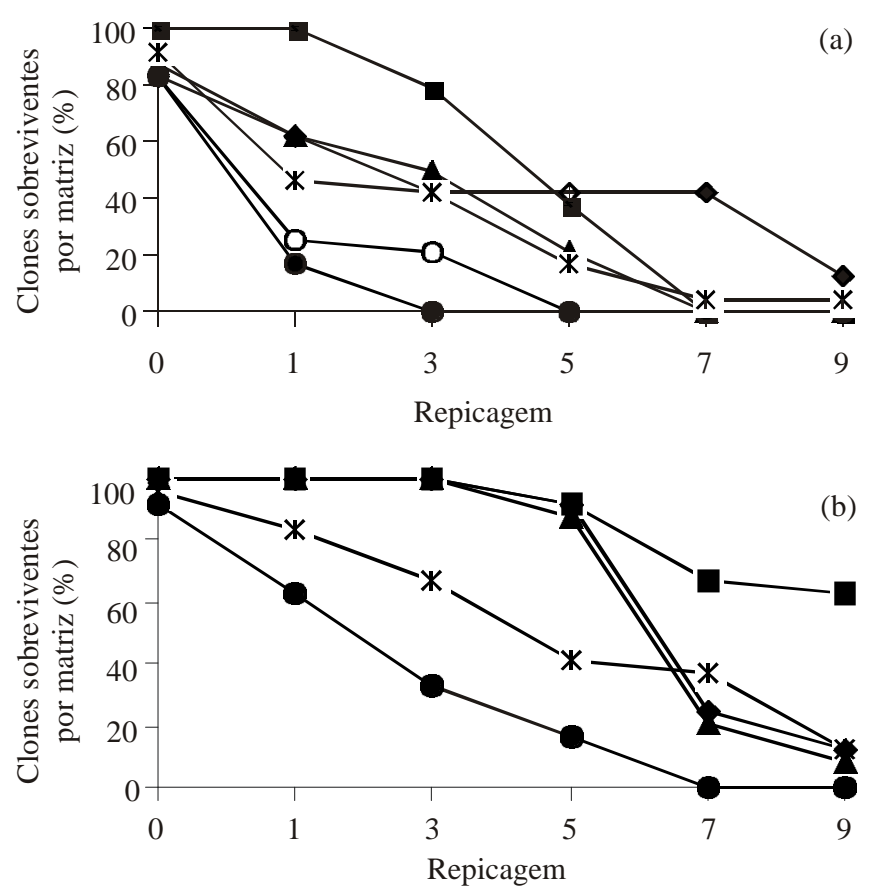

Figura 1. Porcentagem de clones sobreviventes provenientes das matrizes de mangabeira A1 ( $), \mathrm{P} 1(\boldsymbol{\Delta}), \mathrm{P} 8(\mathbf{0}), \mathrm{P} 12(\boldsymbol{\square})$, P13 (•) e G1 (*) no processo de multiplicação de 9 repicagens (a); porcentagem de clones sobreviventes provenientes das

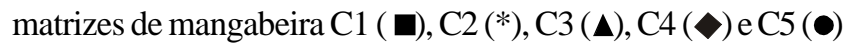
no processo de multiplicação com 9 repicagens (b). 
dentro da matriz (Tabela 1). Nenhum clone da matriz P13 sobreviveu e as matrizes C1, C3 e C4 apresentaram $100 \%$ de sobrevivência dos clones obtidos (Figura 1b). Quanto ao desempenho, as matrizes foram separadas em dois grupos: o primeiro, contendo as matrizes P12, C1, C3, C4, C2, P1 e G1, e o segundo grupo, contendo as matrizes C5, A1, P8 e P13 (Tabela 2). Nenhum clone da matriz P13 sobreviveu nesta repicagem.

$\mathrm{Na}$ quinta repicagem, houve diferença significativa entre as matrizes, atribuindo-se $32 \%$ da variabilidade à matriz e $68 \%$ ao clone dentro da matriz (Tabela 1). Houve aumento de $11 \%$ na variabilidade atribuída à matriz, quando comparada à terceira repicagem, conseqüentemente diminuindo a variabilidade do clone. A variação entre as matrizes na porcentagem de sobrevivência nesta avaliação continuou diminuindo; todos os clones das matrizes P13 e P8 não sobreviveram e as matrizes $\mathrm{C} 1$, $\mathrm{C} 4$ e $\mathrm{C} 3$ continuaram sendo as com maior porcentagem de sobrevivência nesta repicagem (Figura 1).

Houve diferença significativa entre as matrizes, podendo-se separá-las em dois grupos de acordo com o desempenho; o primeiro, contendo as matrizes $\mathrm{C} 1, \mathrm{C} 3$, $\mathrm{C} 4$ e $\mathrm{C} 2$ e, o segundo, contendo as matrizes P12, A1, P1, C5, G1, P8 e P13, em ordem decrescente de desempenho (Tabela 2).

$\mathrm{Na}$ análise de variância da sétima multiplicação dos explantes, houve diferença significativa entre as matrizes, podendo-se inferir que $19 \%$ da variabilidade ocorreu em virtude da matriz e $81 \%$, do clone dentro da matriz (Tabela 1). As matrizes P1, P8, P12, P13 e C5 não sobreviveram nesta repicagem (Figura 1a). Nas avaliações anteriores, sempre duas ou três matrizes se destacavam; nesta avaliação, a matriz $\mathrm{C} 1$, pela primeira vez, se destacou entre as outras matrizes, com $66,7 \%$ de sobrevivência dos clones.

Pode-se separar as matrizes em dois grupos diferenciados de desempenho; o primeiro contendo as matrizes C1, A1, C2, C4, C3 e G1, com os melhores resultados em ordem decrescente (Tabela 2). O segundo grupo contém as matrizes em que todos os clones não sobreviveram ao processo de multiplicação ( $\mathrm{P} 1, \mathrm{P} 8, \mathrm{P} 12, \mathrm{P} 13$ e C5).

Na nona e última repicagem dos explantes, houve diferença significativa entre as matrizes, mas apenas $12 \%$ da variabilidade foi atribuída à matriz e $88 \%$ ao clone dentro da matriz. Estes resultados são semelhantes aos obtidos na sétima repicagem (Tabela 1).
A variação na porcentagem de sobrevivência das matrizes nesta nona repicagem continuou diminuindo, $\mathrm{e}$ todos os clones das matrizes P1, P8, P12, P13 e C5 não sobreviveram (Figura 1a). Por sua vez, a matriz C1, com $62,5 \%$ de sobrevivência dos clones, manteve lugar de destaque até a última avaliação da repicagem. Foi observada uma diferença de desempenho de no mínimo $50 \%$ entre a matriz $\mathrm{C} 1 \mathrm{e}$ as demais matrizes nesta última avaliação.

Com base na análise não-paramétrica, as matrizes foram separadas em dois grupos diferenciados de desempenho; o primeiro, contendo as matrizes $\mathrm{C} 1, \mathrm{C} 2, \mathrm{C} 4$, e A1, em que foram obtidos os melhores resultados em ordem decrescente (Tabela 2). O segundo grupo contém as matrizes nas quais nenhum clone sobreviveu ao processo de multiplicação, e duas matrizes com clones até a última repicagem (C3 e G1).

Segundo Melo et al. (1998), para se obter uma grande variabilidade, é necessário coletar pequenas quantidades de sementes, em diferentes matrizes, que devem ser selecionadas para coleta com certo afastamento entre si, para não haver redução da variabilidade. Grigoletto (1997) verificou que, além do clone, a matriz também pode influenciar nas respostas dos seus novos genótipos.

Neste trabalho, em todo o processo de multiplicação, a maior variabilidade obtida foi entre os clones dentro da matriz. A matriz influenciou seus novos genótipos, mas a maior variabilidade é proveniente do clone, o que mostra a importância da seleção de clones na multiplicação in vitro da mangabeira. No processo de multiplicação dos clones, a partir da sétima repicagem houve, para a maioira dos clones, baixa porcentagem de sobrevivência, com exceção daqueles provenientes da matriz $\mathrm{C} 1$.

A matriz $\mathrm{C} 1$ obteve melhor desempenho, tanto na germinação, como em todo processo de multiplicação e entre os vinte e sete clones provenientes das diferentes matrizes, o número 15 da matriz $\mathrm{C} 1$ foi o que obteve maior quantidade de explantes, totalizando 1.275 explantes na nona repicagem (Tabela 3). Este clone apresentou potencial de produção de mudas de mangabeira in vitro, em larga escala. Por sua vez, clones provenientes desta mesma matriz, como os de número 3,18 e 23 , produziram as menores quantidades de explantes (entre 4 e 9) em nove repicagens.

Portanto, a seleção de clones de mangabeira in vitro deve ser feita dentro das matrizes e até sete repicagens. 
Tabela 3. Número de explantes por clone, obtidos na germinação e nas repicagens de sementes de mangabeira, originárias das matrizes estudadas.

\begin{tabular}{lrlrrrrr}
\hline Matriz & Clone & Germinação & \multicolumn{5}{c}{ Repicagem } \\
\cline { 4 - 8 } & & & $1^{\text {a }}$ & $3^{\text {a }}$ & $5^{\text {a }}$ & 7 a & 9 a \\
\hline C1 & 15 & 1 & 4 & 4 & 24 & 191 & 1.275 \\
C2 & 5 & 1 & 4 & 16 & 16 & 71 & 658 \\
C1 & 11 & 1 & 4 & 4 & 4 & 33 & 574 \\
C1 & 1 & 1 & 4 & 4 & 36 & 117 & 447 \\
C1 & 8 & 1 & 4 & 4 & 28 & 139 & 390 \\
C4 & 23 & 1 & 4 & 4 & 4 & 11 & 385 \\
C1 & 24 & 1 & 4 & 4 & 20 & 103 & 256 \\
C4 & 3 & 1 & 4 & 4 & 8 & 84 & 253 \\
C2 & 6 & 1 & 4 & 12 & 8 & 26 & 170 \\
C1 & 4 & 1 & 4 & 4 & 16 & 44 & 166 \\
G1 & 2 & 1 & 4 & 20 & 12 & 70 & 163 \\
C3 & 13 & 1 & 4 & 4 & 48 & 13 & 140 \\
C1 & 5 & 1 & 4 & 4 & 24 & 44 & 135 \\
A1 & 23 & 1 & 4 & 4 & 8 & 17 & 120 \\
C3 & 16 & 1 & 4 & 4 & 20 & 14 & 96 \\
C1 & 13 & 1 & 4 & 4 & 4 & 4 & 69 \\
C1 & 16 & 1 & 4 & 4 & 12 & 16 & 46 \\
A1 & 16 & 1 & 4 & 4 & 8 & 5 & 40 \\
C1 & 7 & 1 & 4 & 4 & 12 & 68 & 33 \\
C1 & 10 & 1 & 4 & 4 & 8 & 12 & 30 \\
C1 & 21 & 1 & 4 & 4 & 4 & 5 & 25 \\
C4 & 24 & 1 & 4 & 4 & 4 & 4 & 23 \\
C2 & 3 & 1 & 4 & 12 & 12 & 6 & 18 \\
C1 & 3 & 1 & 4 & 4 & 4 & 4 & 9 \\
C1 & 18 & 1 & 4 & 4 & 8 & 8 & 8 \\
C1 & 23 & 1 & 4 & 4 & 16 & 4 & 4 \\
A1 & 11 & 1 & 4 & 4 & 4 & 1 & 4 \\
\hline & & & & & & &
\end{tabular}

A partir desta, a taxa de mortalidade dos clones, com potencial de multiplicação, se mantém constante.

\section{Conclusões}

1. Não há diferença significativa entre as matrizes testadas.

2. A fim de selecionar os melhores clones para produção in vitro de mudas de mangabeira, deve-se realizar até a sétima repicagem.

3. A melhor matriz selecionada é a C1 e o melhor clone para produção in vitro de mudas de mangabeira em larga escala é o C1 15.

\section{Referências}

ANDRADE, L.R.M.; PEREIRA, A.V.; JUNQUEIRA, N.T.V.; SILVA, J.A. da; SILVA, D.B. da. Fertilização de substrato para mudas de mangabeira (Hancornia speciosa Gomez). Planaltina: Embrapa-CPAC, 1999. 3p. (Pesquisa em Andamento, 24).

FERREIRA, M.B. Plantas de substâncias medicamentosas, de uso popular, nos cerrados de Minas Gerais. Informe Agropecuário, v.6, p.19-23, 1980.

GRIGOLETTO, E.R. Micropropagação de Hancornia speciosa Gómez (mangabeira). 1997. 68p. Dissertação (Mestrado) Universidade de Brasília, Brasília.

HIRSCHMANN, G.S.; ARIAS, A.R. de. A survey of medicinal plants of Minas Gerais, Brazil. Journal of Ethnopharmacology, v.29, p.159-172, 1990.

JUNQUEIRA, N.T.V.; SILVA, J.A.; CHARCHAR, M.J.d'A.; ANDRADE, L.R.M. Controle integrado da podridão de raízes (Cylindrocladium clavatum) de mudas de mangabeira. In: EMBRAPA. Centro de Pesquisa Agropecuária dos Cerrados (Planaltina, DF). Relatório Técnico Anual do Centro de Pesquisa Agropecuária dos Cerrados: 1991 a 1995. Planaltina, 1997. p.202-203.

MELO, J.T. de. Respostas de mudas de espécies arbóreas do cerrado a nutrientes em Latossolo Vermelho Escuro. 1999. 104p. Tese (Doutorado) - Universidade de Brasília, Brasília.

MELO, J.T.; SILVA, J.A.; TORRES, A.R.A.; SILVEIRA, C.E.S.; CALDAS, L.S. Coleta, propagação e desenvolvimento inicial de espécies do cerrado. In: SANO, S.M.; ALMEIDA, S.P. Cerrado: ambiente e flora. Planaltina: Embrapa-CPAC, 1998. p.195-243.

MUNIZ, M. de F.S.; MELO, M.M.P. de; QUEIROZ, F.M.; MENEZES, M.; CASTRO, N.R. Colletotrichum gloeosporioides como patógeno da mangabeira (Hancornia speciosa) no Brasil. Summa Phytopathologica, v.23, p.42-45, 1997.

PARENTE, T.V.; BORGO, L.A.; MACHADO, J.W.B. Características físico-químicas de frutos de mangaba de cerrado da região geoeconômica do Distrito Federal. Ciência e Cultura, v.37, p.95-98, 1985.

PAULA, J.E. de. Cerrado: sugestão para a adequação entre produção e preservação. Informe Agropecuário, v.16, p.1-2, 47-48, 1992.

PEREIRA, A.V.; PEREIRA, E.B.C.; JUNQUEIRA, N.T.V. Propagação e domesticação de plantas nativas do cerrado com potencial econômico. Horticultura Brasileira, v.19, 2001. Suplemento. CD-ROM.

SAS INSTITUTE INC. (Cary, Estados Unidos). SAS statistical analysis system: user's guide. Cary, 1985. 295p.

WOLF, D.A.; HOLLANDER, M. Nonparametric statistical methods. New York: J. Wiley, 1973. 503p. 